\title{
STABILIZING A BLASIUS BOUNDARY LAYER WITH DIELECTRIC BARRIER DISCHARGE PLASMA ACTUATION: EXPERIMENTAL CHARACTERIZATION
}

\section{N. Szulga, O. Vermeersch, M. Forte, and G. Casalis}

ONERA, The French Aerospace Lab.

Centre de Toulouse

2 Av. Edouard Belin, Toulouse 31055, France

\begin{abstract}
This paper presents an experimental characterization of Dielectric Barrier Discharge (DBD) plasma actuation on a two-dimensional (2D) boundary layer around a flat plate using both Laser Doppler Anemometry (LDA) and hot wire probings. The experiments are conducted in a subsonic wind tunnel at a free-stream velocity $U_{\infty}=35 \mathrm{~m} / \mathrm{s}$ for a single DBD actuator and several electrical parameters. Hot wire probings are used to quantify the transition delay and mean velocity profiles far enough from the plasma region, while mean velocity profile inside the plasma extent are measured using LDA after the method has been validated from a comparison with hot wire measurements. Measurements inside the plasma extent allow to quantify the ionic wind contribution to the actuated mean velocity profiles in a case where the transition is delayed by the actuator. A maximal ionic wind of $7 \mathrm{~m} / \mathrm{s}$ is found to be added $2 \mathrm{~mm}$ downstream the actuator for a consumed electrical power of $80 \mathrm{~W} / \mathrm{m}$.
\end{abstract}

\section{INTRODUCTION}

One possible way to reduce aircraft fuel consumption is to delay the boundary layer transition around wings in order to decrease skin friction drag. Stabilizing the boundary layer mean velocity profiles is a possible approach to achieve this aim. Among the possible techniques, plasma actuation turns out to be an interesting solution because of its easy implementation on a surface, the light weight of the actuators, and their input energy (electricity) which allows easy control and modulation. In this work, the actuation is performed using a DBD plasma actuator. This type of actuator is constituted of two electrodes stuck on each face of a dielectric material. When an alternative high voltage is applied between

(C) The authors, published by EDP Sciences. This is an Open Access article distributed under the terms of the Creative Commons Attribution License 4.0 (http://creativecommons.org/licenses/by/4.0/). 
the electrodes, the ambient air is ionized. The charged particles drift under the effect of the electric field and create a body force tangential to the wall (the ionic wind), which is used in this case for transition control. The experiments on transition delay have been successfully conducted for $2 \mathrm{D}$ configurations on a flat plate $[1,2]$ and on a wing profile $[3,4]$ for velocities of 20 and $35 \mathrm{~m} / \mathrm{s}$, respectively.

The first set of experiments has been made previously on an ONERA-D $2 \mathrm{D}$ profile [5]. In this case, the transition was induced by Tollmien-Schlichting (T-S) waves. Mean velocity profiles were measured outside the plasma region and probings along the chord were performed. The maximum transition delay of $6 \%$ of chord has been observed for a free-stream velocity $U_{\infty}=21 \mathrm{~m} / \mathrm{s}$. The first analytic model for the tangential component of the body force field has been developed and integrated in the ONERA in-house boundary layer code 3C3D from these measurements. This model is able to represent the mean velocity profiles in the vicinity of the wall but could not compute the effect of plasma actuation near the upper edge of the boundary layer. As a result, the computed transition delay due to plasma actuation was slightly overpredicted.

In this paper, a new set of experiments, conducted on a flat plate and for free-stream velocities higher than $30 \mathrm{~m} / \mathrm{s}$, are presented. Combined LDA and hot wire measurements are performed to assess the effect of DBD plasma actuation on the boundary layer both inside and out of the plasma region. A focus is made on the mean body force effect, also called steady actuation on the delay of a T-S induced transition. This new set of measurements will allow to improve the existing body force model.

\section{EXPERIMENTAL SETUP}

The experiments are conducted in the TRIN 2 subsonic open-return research wind tunnel located at ONERA Toulouse. It features a low turbulence level $\left(1 \cdot 10^{-3} \leq \mathrm{Tu} \leq 2 \cdot 10^{-3}\right)$ and a free-stream velocity range of $25 \leq U_{\infty} \leq 50 \mathrm{~m} / \mathrm{s}$, which make it well suited to laminarity and transition studies. The experiments are performed on a flat plate set at a slightly positive angle of attack (AoA $\left.=0.13^{\circ}\right)$. The plate is $1.175 \mathrm{~mm}$ long and $40 \mathrm{~cm}$ wide. It is equipped with a dielectric insert made of Polymethyl methacrylate, which enables to outfit the model with the desired number of actuators between 370 and $820 \mathrm{~mm}$ downstream the leading edge. In the present case of study, a single DBD actuator is set at $x_{\mathrm{DBD}}=470 \mathrm{~mm}$ from the leading edge as shown in Fig. 1. Both electrodes are $21 \mathrm{~mm}$ in width and their spanwise length is $150 \mathrm{~mm}$. A 2-millimeter gap separates the electrodes. Below, in Figs 2, 3, and 5, the dielectric insert is represented by a lighter segment on the line parallel to the $x$-axis.

The air-exposed electrode is connected to a voltage amplifier (Trek, model $30 / 20 \mathrm{~A}$, gain $3000 \mathrm{~V} / \mathrm{V}$ ), while the other is grounded. The input signal has a sinusoidal waveform. The electric power consumed by a plasma actuator is 
computed from current and voltage measurements following the relation:

$$
P=\frac{1}{T} \int_{0}^{T} V(t) i(t) d t .
$$

A high frequency/current transformer (Magnelab, model CT-D0.5, sensibility $0.5 \mathrm{~V} / \mathrm{A}$, bandwidth $48 \mathrm{~Hz}-$ $200 \mathrm{MHz}$ ) measures the instantaneous discharge current of the actuator $i(t)$,

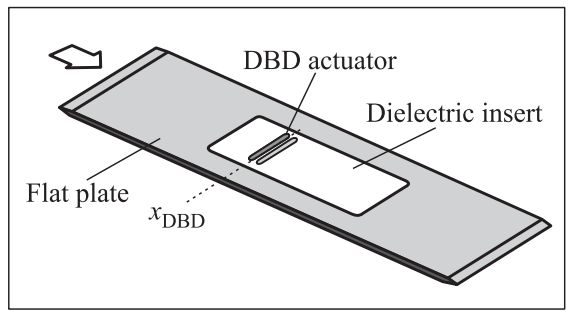

Figure 1 Implementation of the DBD actuator on the flat plate while $V(t)$ is the instantaneous input voltage given by the monitoring sensor of the amplifier. The average on a sufficiently large number of periods $T$ of $V(t) i(t)$, computed by a digital oscilloscope (Lecroy wavesurfer 454 bandwidth $500 \mathrm{MHz}$ ) gives the mean consumed power $P$. This value is then divided by the spanwise length of the electrodes to give the consumed power per unit of electrode length $P / L$ in $\mathrm{W} / \mathrm{m}$. The voltage amplitude varies between 9 and $10.8 \mathrm{kV}$ and the input frequency is set at $3 \mathrm{kHz}$, resulting in a consumed power range between 40 and $80 \mathrm{~W} / \mathrm{m}$.

The measurements into the boundary layer are carried out using hot-wire anemometry (Dantec Streamline, 90C10 CTA module, 55P15 probes) and LDA for different configurations with and without control. For the hot wire anemometry, the acquisition is performed over 80000 samples. The signal from the anemometer is low-pass filtered at $8 \mathrm{kHz}$ in order to avoid aliasing and amplified 10 times so as to benefit from the best available resolution of the Ana$\log$ /Digital board. The sampling rate $(20 \mathrm{kHz})$ is chosen to satisfy the NyquistShannon theorem. The raw signal is acquired simultaneously without filtering so as to record the mean value. The laser used for LDA is an Oxxius Laser (wavelength $532 \mathrm{~nm}$ and power $300 \mathrm{~mW}$ ). The emission optics has a 0.46 -meter focal length. The DISA 55X optic reception is mounted in a forward scattered mode. Seeding is realized using DEHS (di-ethyl-hexyl-sebacat) mineral oil. The measuring volume is about $100 \mu \mathrm{m}$ in its smaller diameter and $3 \mathrm{~mm}$ in its greatest diameter. The optical signal is processed by a digital burst correlator TSI IFA 655 .

The measurements were conducted for a free-stream velocity $U_{\infty}=35 \mathrm{~m} / \mathrm{s}$.

\section{BASELINE FLOW}

As the flat plate is not equipped with pressure taps, the pressure distribution is determined from the hot wire measurements. Mean velocity profiles are measured along the flat plate and the boundary layer thickness is assessed for each mean 


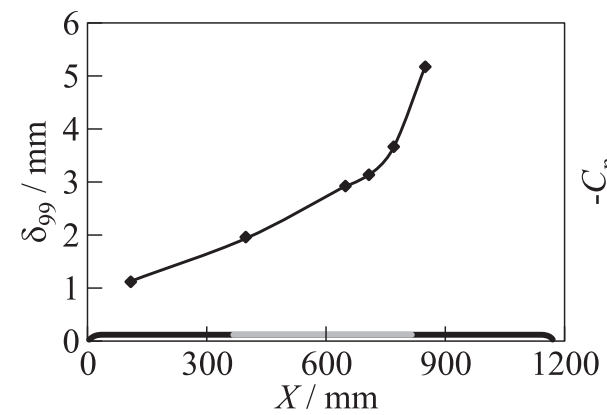

(a)

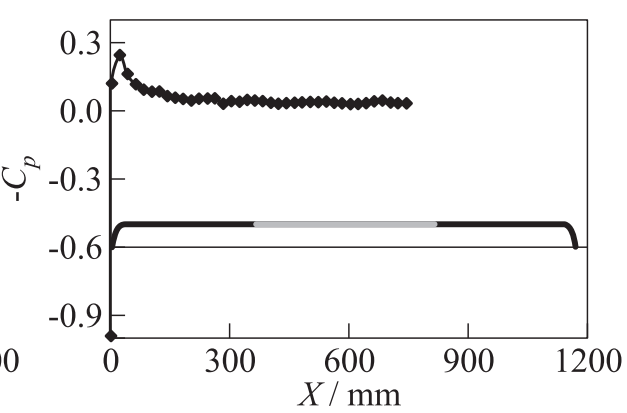

(b)

Figure 2 Boundary layer thickness evolution along the plate for $U_{\infty}=35 \mathrm{~m} / \mathrm{s}(a)$ and measured pressure coefficients $(b)$

velocity profile. In particular, the boundary layer thickness evolution along the flat plate is given Fig. $2 a$. The pressure coefficient is quantified from free-stream velocity measurements using hot wire anemometry. The resulting evolution of the pressure coefficient is presented Fig. $2 b$. It features a suction peak followed by a large and weak deceleration, featuring a pressure gradient close to 0 , which causes the destabilization of the T-S waves. This pressure coefficient distribution is extrapolated in order to allow one to perform boundary layer and stability computations.

The transition location is determined by measuring the hot wire root mean square (RMS) signal along the plate length at a constant height from the wall $(z=1 \mathrm{~mm})$. The tangents of the RMS signal in the laminar and the intermittent phases are then traced (black dotted line in Fig. 3). Their intersection gives the transition position. Figure 3 shows that the natural transition location for $U_{\infty}=35 \mathrm{~m} / \mathrm{s}$ is at $x_{\mathrm{tr}}=670 \mathrm{~mm}$. This is about $3 \mathrm{~cm}$ before the boundary

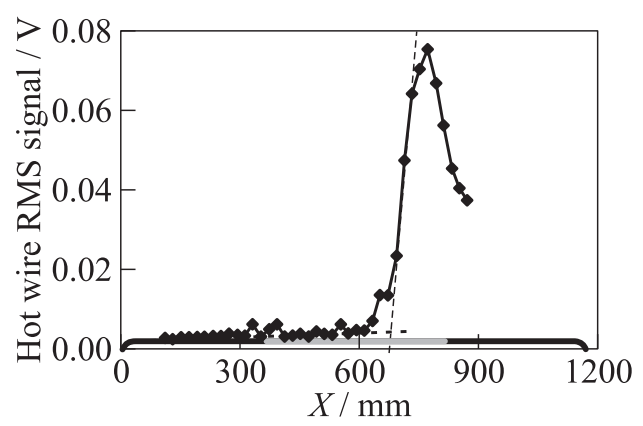

Figure 3 Hot wire RMS signal along the flat plate for $U_{\infty}=35 \mathrm{~m} / \mathrm{s}$ 


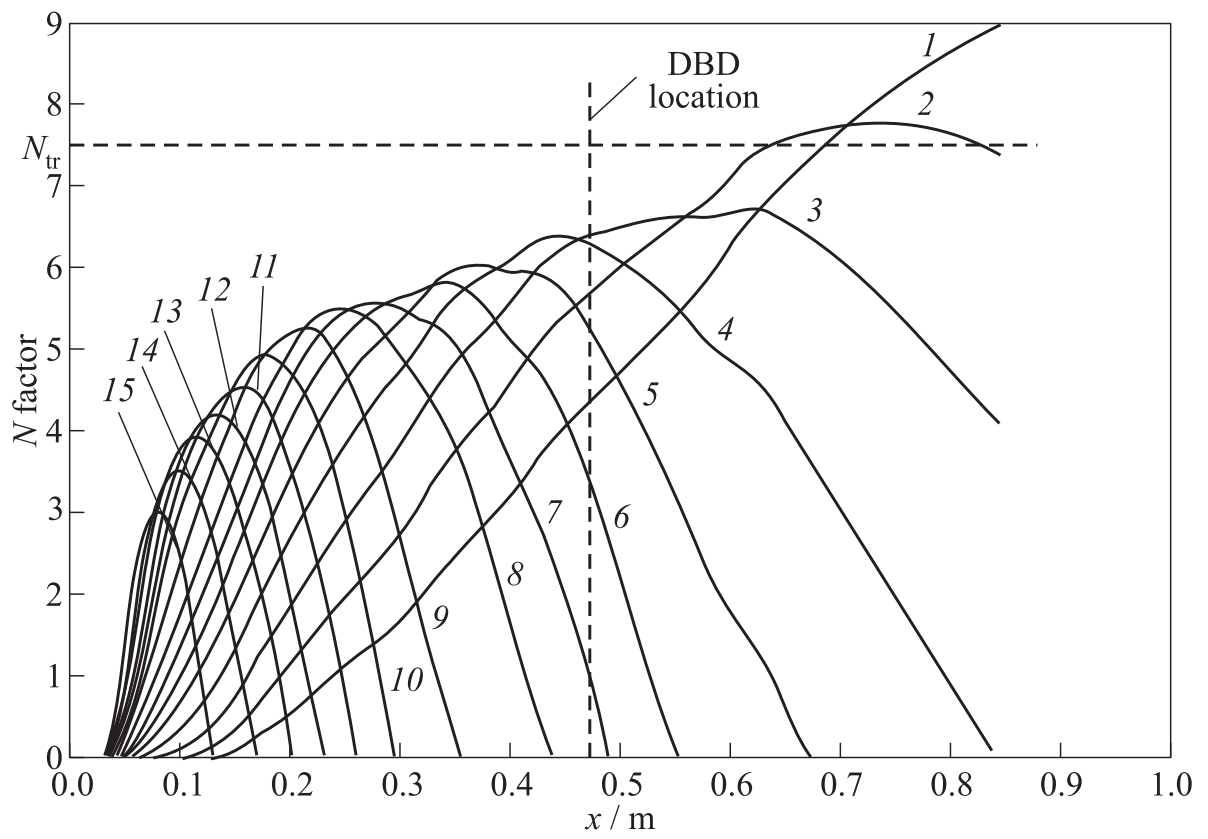

Figure 4 Evolution of T-S waves amplification along the flat plate for $U_{\infty}=35 \mathrm{~m} / \mathrm{s}$ : $1-500 \mathrm{~Hz} ; 2-600 ; 3-700 ; 4-800 ; 5-900 ; 6-1000 ; 7-1100 ; 8-1200$; $9-1400 ; 10-1600 ; 11-1800 ; 12-2000 ; 13-2200 ; 14-2500$; and $15-$ $3000 \mathrm{~Hz}$

thickens due to a turbulent regime, as seen in Fig. $2 a$. The actuation is performed into the laminar zone.

As the DBD actuator induces a pulsed body force at the same frequency $f_{p}$ as the input signal [6], it may excite the corresponding T-S waves and trigger a promoted transition if $f_{p}$ is not well chosen [5]. For this reason, boundary layer mean velocity profiles have been computed from the experimental pressure distribution using an ONERA in-house boundary layer code (3C3D) and linear stability has then been applied. This results in the $N$-factor chart of Fig. 4 which describes the evolution of the T-S waves amplification along the flat plate. In the present case, the turbulence level is $\mathrm{Tu} \approx 0.13 \%$ which corresponds to a transition $N$-factor $N_{\mathrm{tr}} \approx 7.5$ according to Mack relation $\left(N_{\mathrm{tr}}=-8.43-2.4 \ln (\mathrm{Tu})\right)[7]$. The T-S waves frequency which triggers the transition is $f_{\mathrm{tr}}=600 \mathrm{~Hz}$. At the actuator location $\left(x_{\mathrm{DBD}}=470 \mathrm{~mm}\right)$, T-S waves at frequencies below $700 \mathrm{~Hz}$ are amplified whereas the others are damped. To avoid any excitation of the T-S waves by the actuation and to focus on a steady actuation, the input signal frequency must be chosen sufficiently higher than the local T-S waves frequencies. Thus, it is set at $3 \mathrm{kHz}$. 


\section{MEASUREMENTS OUTSIDE THE PLASMA EXTENT}

\subsection{Transition Measurements}

The transition position is determined using the method described in section 3 and illustrated in Fig. 3.

Figure 5 shows the evolution of a hot wire probe RMS signal along the plate for a boundary layer submitted to DBD plasma actuation at two different consumed powers: $P / L=40$ and $80 \mathrm{~W} / \mathrm{m}$. The input signal frequency is set at $3 \mathrm{kHz}$ for both cases. For $U_{\infty}=35 \mathrm{~m} / \mathrm{s}$ and both consumed power, the actuation results in a transition delay of at least $30 \mathrm{~mm}$. Transition delay could not be measured for a consumed power of $90 \mathrm{~W} / \mathrm{m}$. More-

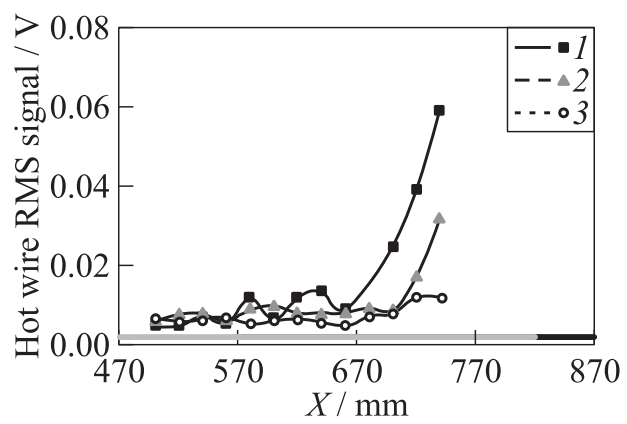

Figure 5 Hot wire RMS signal at a constant height $(z=1 \mathrm{~mm})$ along the plate for $U_{\infty}=35 \mathrm{~m} / \mathrm{s}$ ( 1 - baseline $)$ and two different actuation cases $(2-P / L=40 \mathrm{~W} / \mathrm{m}$ and $3-P / L=80 \mathrm{~W} / \mathrm{m})$

over, the transition delay is greater when the consumed electrical power increases. In this study, the focus is not set on optimizing the transition delay obtained with the actuator, but to characterize experimentally the influence of steady DBD actuation on a boundary layer flow. Nonetheless, knowing that the transition is delayed confirms that the chosen input signal frequency does not trigger any unwanted unsteady effect.

\subsection{Mean Velocity Profiles Measurements}

Hot wire anemometry is a classical and reliable method to measure mean velocity profiles inside a boundary layer. However, it has to be used far enough from the plasma extent in order to avoid issues due to charges created by the actuator or by the generated electrical field. To ensure that the described LDA measurements are well resolved for configurations with and without actuation, mean velocity profiles outside the plasma extent are measured using both LDA and hot wire anemometry at corresponding locations.

Figure 6 compares the mean velocity profile measured using LDA and hot wire anemometry without actuation at $x=650 \mathrm{~mm}$ from the plate leading edge. In the configuration without actuation, the mean velocity profile measured with LDA is in good agreement with the one measured with hot wire. 
Mean velocity profiles measured for two different consumed power $(P / L=40$ and $80 \mathrm{~W} / \mathrm{m})$ and using both techniques are also compared. The measurements are performed 50 and $100 \mathrm{~mm}$ downstream the actuator. The resulting mean velocity profiles are represented in Fig. 7 at $x_{\mathrm{DBD}}$ $+50 \mathrm{~mm}$ and in Fig. 8 at $x_{\mathrm{DBD}}$ $+100 \mathrm{~mm}$. For each case with and without actuation, the measured velocity profiles are compared with the corresponding Blasius one.

Figures $7 a$ and $8 a$ show mean velocity profiles without actuation measured, respectively, 50 and $100 \mathrm{~mm}$

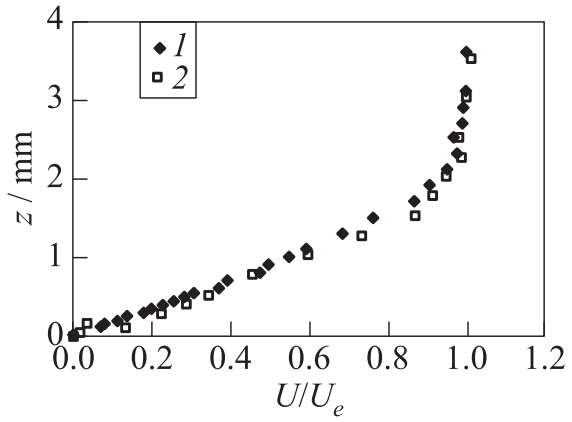

Figure 6 Mean velocity profile measured at $x=650 \mathrm{~mm}$ from the leading edge with a hot wire probe (1) and LDA (2) at $U_{\infty}$ $=35 \mathrm{~m} / \mathrm{s}$ downstream the actuator using hot wire anemometry. At these locations, the boundary layer is still laminar and the mean velocity profile fits well to the corresponding Blasius mean velocity profile.

Figures $7 b$ and $7 c$ show the effect of DBD actuation at $x_{\mathrm{DBD}}+50 \mathrm{~mm}$ for $P / L=40$ and $80 \mathrm{~W} / \mathrm{m}$, respectively. At this station and for both actuation cases, the effect of ionic wind is slightly visible over the entire velocity profile. The ionic wind addition results in fuller profiles for both actuation cases. The boundary layer thickness is not affected by the actuation. At this station, it was found a great accordance between the velocity profiles measured with LDA and with hot wire anemometry.

Figures $8 b$ and $8 c$ show the effect of DBD actuation at $x_{\mathrm{DBD}}+100 \mathrm{~mm}$ for $P / L=40$ and $80 \mathrm{~W} / \mathrm{m}$, respectively. The effect of DBD actuation is barely visible on the mean velocity profiles measured using hot wire anemometry. At this station, there is more discrepancy between the LDA and hot wire measurements, especially close to the wall. Laser Doppler anemometry seems to overestimate the contribution of ionic wind in comparison to the hot wire measurements.

As the boundary layer thickness $\delta$ does not change with actuation, a computation of the displacement thickness $\delta_{1}=\int_{0}^{\delta}\left(1-U(z) / U_{e}\right) d z$ (Table 1) is performed to compare the mean velocity profiles "fullness." This computation is made for the mean velocity profiles measured with hot wire anemometry because of their better accuracy.

The displacement thickness $\delta_{1}$ decreases for an actuated boundary layer. This value can be seen as the distance of a mean velocity profile to the (nonrealistic) mean velocity profile where the velocity would be equal to $U_{e}$ whatever the distance from the wall. The smaller $\delta_{1}$ is, the "fuller" the mean velocity profile. In Table 1, the contribution of ionic wind which makes the mean velocity profiles 


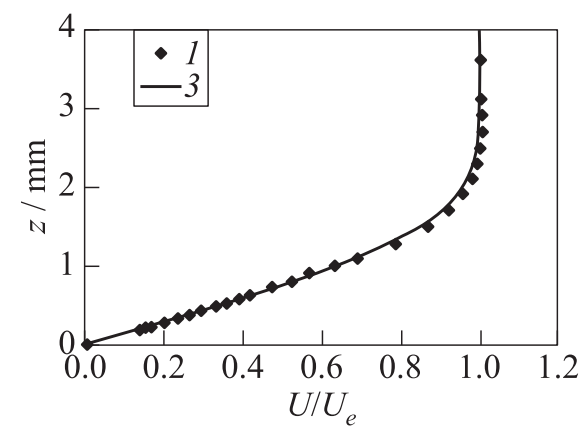

(a)

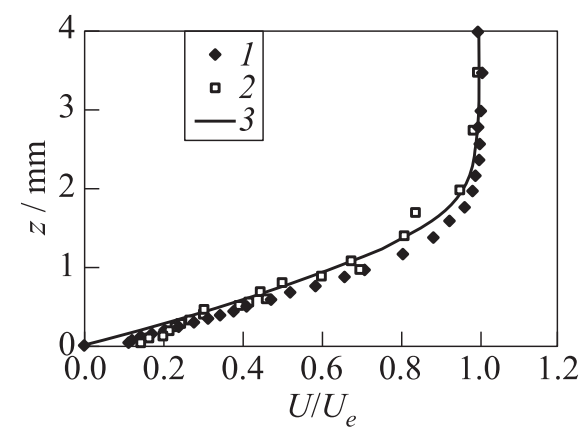

(b)

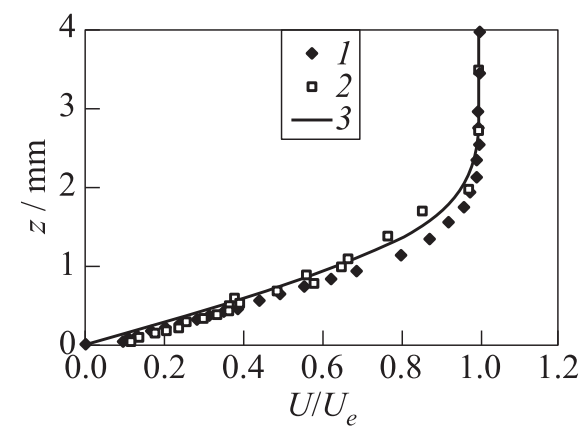

(c)

Figure 7 Mean velocity profiles measured at $x_{\mathrm{DBD}}+50 \mathrm{~mm}(1-$ hot wire and 2 - LDA) compared to the corresponding Blasius velocity profiles (3) for various cases: without actuation $(a)$ and with actuation for $P / L=40(b)$ and $80 \mathrm{~W} / \mathrm{m}(c)$

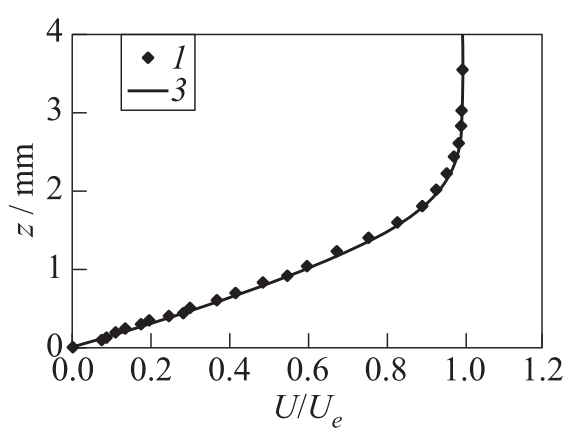

(a)

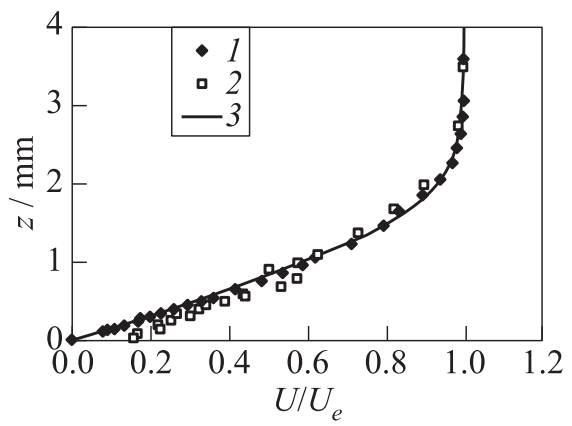

(b)

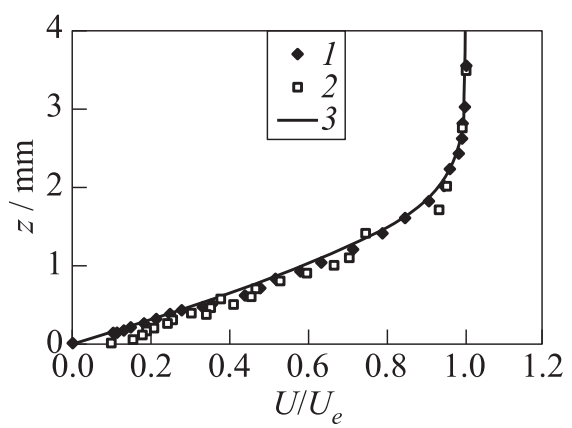

(c)

Figure 8 Mean velocity profiles measured at $x_{\mathrm{DBD}}+100 \mathrm{~mm}(1-$ hot wire and $2-\mathrm{LDA}$ ) compared to the corresponding Blasius velocity profiles (3) for various cases: without actuation $(a)$ and with actuation for $P / L=40(b)$ and $80 \mathrm{~W} / \mathrm{m}(c)$ 
Table 1 Comparison of the displacement thickness $\delta_{1}$ (in $\mathrm{mm}$ ) for the measured mean velocity profiles

\begin{tabular}{cccc}
\hline \multicolumn{1}{c}{ Distance } & Baseline & $P / L=40 \mathrm{~W} / \mathrm{m}$ & $P / L=80 \mathrm{~W} / \mathrm{m}$ \\
\hline$x_{\mathrm{DBD}}+50 \mathrm{~mm}$ & 0.8112 & 0.6974 & 0.7075 \\
$x_{\mathrm{DBD}}+100 \mathrm{~mm}$ & 0.9436 & 0.9039 & 0.8693 \\
\hline
\end{tabular}

fuller, can be seen as far as $100 \mathrm{~mm}$ downstream the actuator through this quantity: the more the consumed energy, the "fuller" the velocity profile, and the smaller the displacement thickness.

\section{MEASUREMENTS INSIDE THE PLASMA EXTENT}

Hot wire anemometry is limited to measurements outside and sufficiently far from the plasma extent due to the electrical field which could damage the probe if placed too close to the actuator. To explore the effect of the body force generated by a plasma actuator on the mean velocity profiles inside or close to the plasma extent, LDA is performed.

\subsection{Mean Velocity Profiles}

Figure 9 shows mean velocity profiles measured at several locations inside or close to the plasma extent for different cases with and without actuation. These mean velocity profiles are compared to the corresponding Blasius velocity profile at each location. Each measurement point is shown by the sign corresponding to the particle rate measured at the same point.

For all the measured velocity profiles, the particle rate decreases as the measurement gets closer to the wall. When comparing velocity profiles with and without actuation, a drop in the particle rate is noticeable when the actuator is powered. Moreover, mean velocity profiles measured for the highest consumed power $(P / L=80 \mathrm{~W} / \mathrm{m})$ show a lower particle rate than the mean velocity profiles measured for $P / L=40 \mathrm{~W} / \mathrm{m}$.

Each actuated mean velocity profile (see Figs. $9 b$ and $9 c$ ) shows a noticeable deformation below $z=1 \mathrm{~mm}$ from the wall. As a powered DBD actuator induces an electric field, seeding particles may become charged and then drift under electrical effects when moving through the plasma extent. As a consequence, the observed mean velocity profile changes may be caused both by a mechanical and charged particle effects. In order to know whether particles are charged through the plasma extent, velocity histograms are analyzed. The examples of 


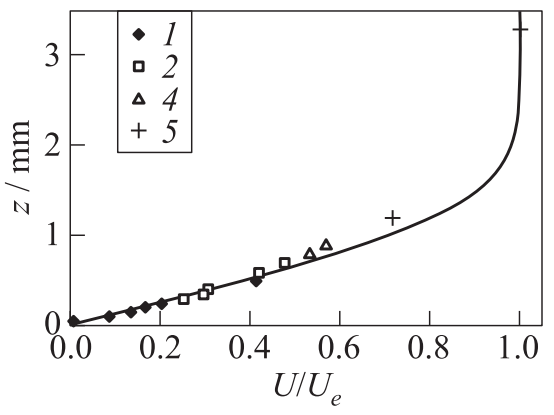

(a)

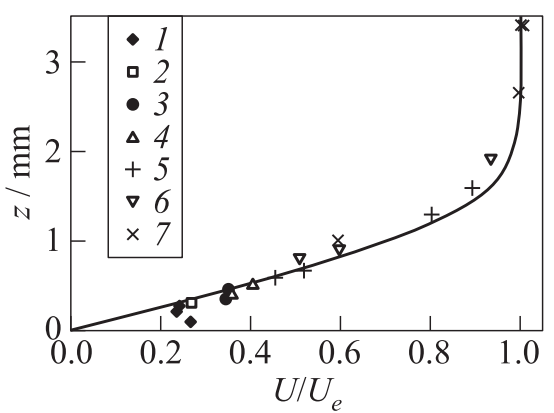

(b)

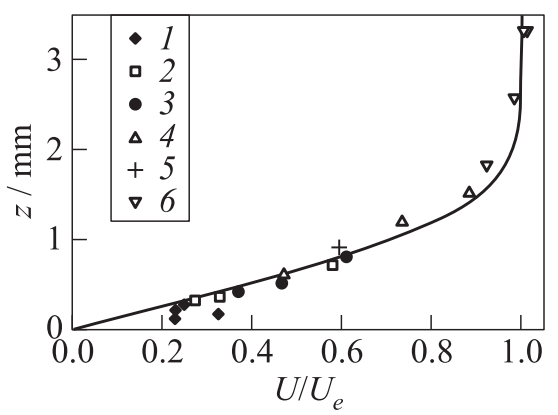

(c)

Figure 9 Mean velocity profiles measured at $x_{\mathrm{DBD}}+2 \mathrm{~mm}$ compared to the corresponding Blasius velocity profile (curve) for various cases: without actuation $(a)$ and with actuation for $P / L=40(b)$ and $80 \mathrm{~W} / \mathrm{m}(c)$ : $1-$ particle rate $=1 \mathrm{kHz} ; 2-5 ; 3-10$; $4-20 ; 5-30 ; 6-40$; and $7-$ particle rate $=50 \mathrm{kHz}$ such histograms are shown in Fig. 10. Figure $10 a$ shows a velocity histogram for a measurement point without actuation. The velocity distribution has a classic gaussian shape. Figure $10 b$ shows a velocity histogram with an actuation at $P / L=80 \mathrm{~W} / \mathrm{m}$ for the same measurement point. With actuation, the velocity histogram has a nonstandard shape which appears to be a combination of two gaussian velocity distributions. Moreover, one of these is centered around the same velocity whatever the measurement point for the same set of electrical parameters (voltage amplitude and frequency). In this case, this second gaussian is centered around a velocity of $29 \mathrm{~m} / \mathrm{s}$ as seen by confronting Figs. $10 b$ and 11 . This may be due to the electrical field affecting the seeding particles. In order to only consider the DBD mechanical effect, this second gaussian distribution is dismissed. The resulting mean velocity profiles are shown in Figs. 12 to 14 for various positions inside or close to the plasma extent.

The mean velocity profiles correction is more visible for the measurements at $x_{\mathrm{DBD}}+2 \mathrm{~mm}$ as featured in Fig. 13. This position corresponds to the upstream edge of the grounded electrode and is inside the plasma extent. Figure 12 shows that an effect of DBD plasma actuation is visible on the mean velocity profiles $2 \mathrm{~mm}$ upstream the plasma extent beginning. 


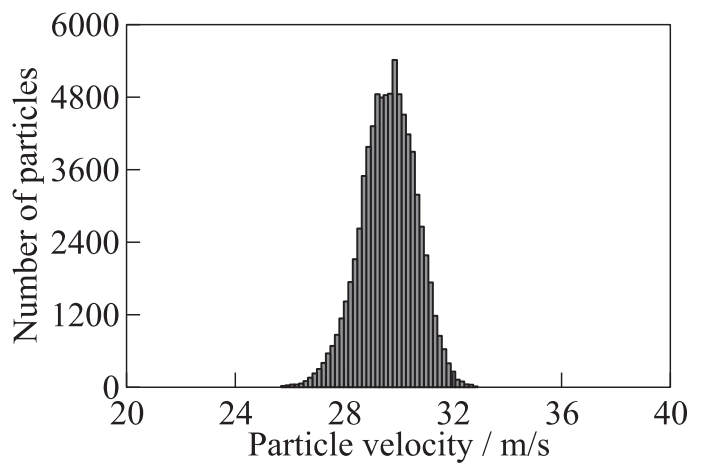

(a)

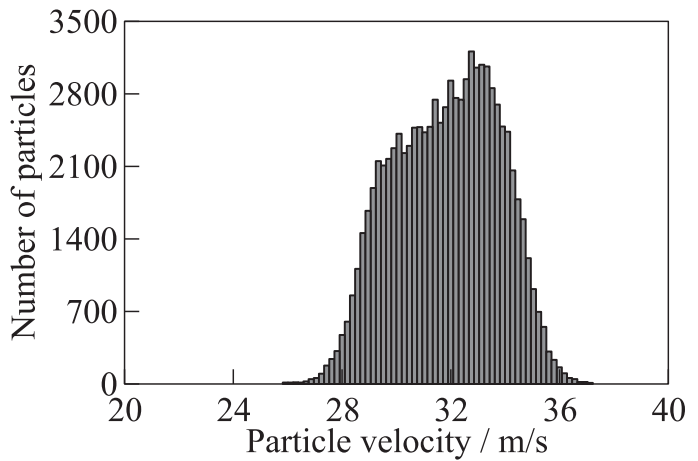

(b)

Figure 10 Velocity histograms of measurement points located at $x_{\mathrm{DBD}}+2 \mathrm{~mm}$ and $Z=0.5 \mathrm{~mm}$ from the wall for various cases: $(a)$ without actuation; and $(b)$ with actuation for $P / L=80 \mathrm{~W} / \mathrm{m}$

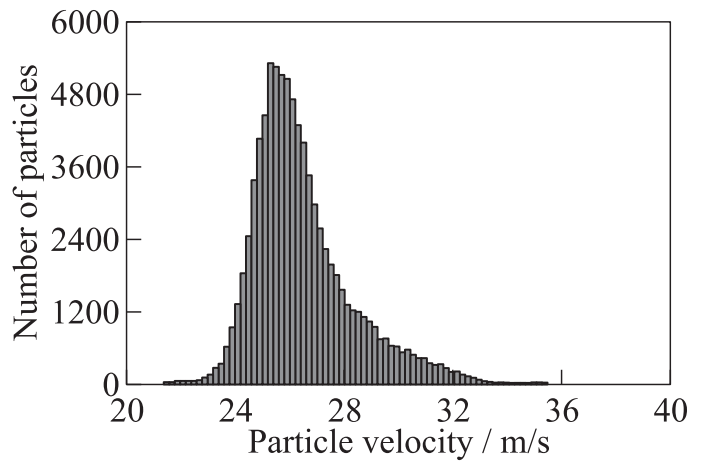

Figure 11 Velocity histograms of the measurement point located at $x_{\mathrm{DBD}}+2 \mathrm{~mm}$ and $Z=0.4 \mathrm{~mm}$ from the wall, DBD on, $P / L=80 \mathrm{~W} / \mathrm{m}$ 


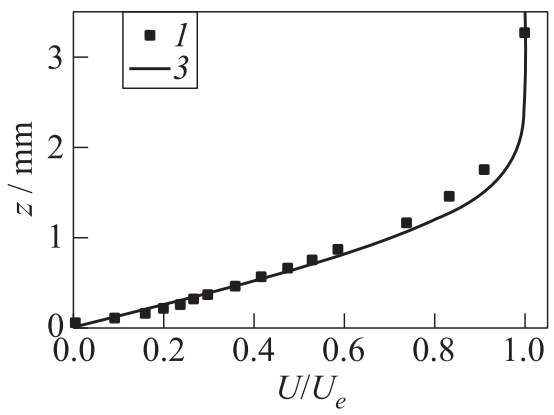

(a)

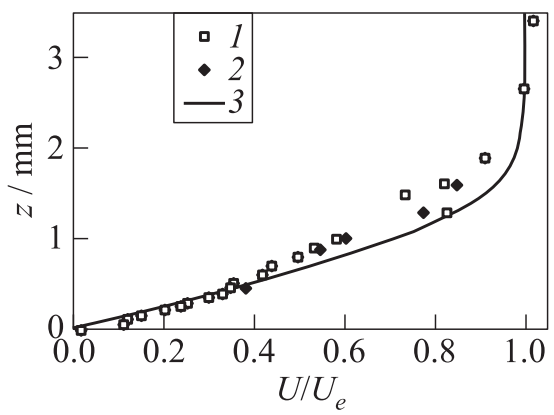

(b)

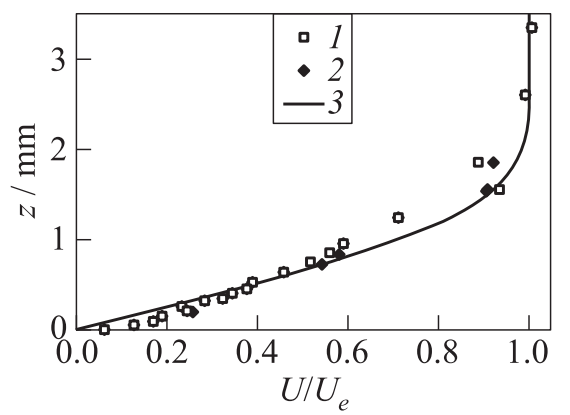

(c)

Figure 12 Mean velocity profiles measured at $x_{\mathrm{DBD}}-2 \mathrm{~mm}(1-$ corrected and 2 - noncorrected) compared to the corresponding Blasius velocity profile (3) for various cases: without actuation $(a)$ and with actuation for $P / L=40(b)$ and $80 \mathrm{~W} / \mathrm{m}(c)$

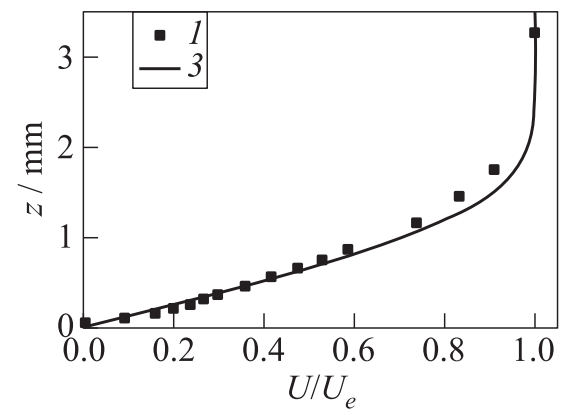

(a)

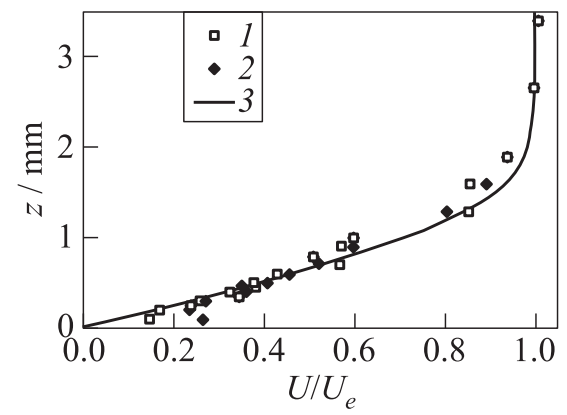

(b)

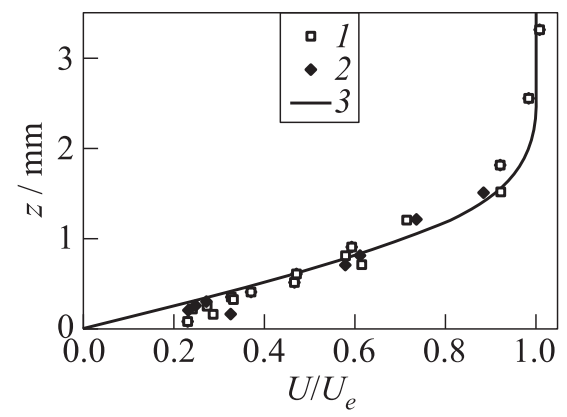

(c)

Figure 13 Mean velocity profiles measured at $x_{\mathrm{DBD}}+2 \mathrm{~mm}(1-$ corrected and 2 - noncorrected) compared to the corresponding Blasius velocity profile (3) for various cases: without actuation $(a)$ and with actuation for $P / L=40(b)$ and $80 \mathrm{~W} / \mathrm{m}(c)$ 


\subsection{Ionic Wind Contribution in the Actuated Mean Velocity Profiles}

In order to quantify the ionic wind contribution in the actuated mean velocity profiles, the added ionic wind is computed by substracting the baseline one to the actuated ones. The mean velocity profiles used for the calculations are those presented in Figs. 12 to 14 . Figures 15 and 16 show the resulting ionic wind contribution evolution inside and close to the plasma extent for the two studied consumed electric powers.

In both actuation cases, Figs. $15 a$ and $16 a$ show that the ionic wind contribution is negative between $Z=0.5$ and $2 \mathrm{~mm}$ from the wall. This may be due to a suction zone occurring downstream the exposed electrode edge as observed by Debien et al. [8]. Figure 15 shows the diffusion of the transferred momentum from the plasma to the boundary layer. This is comparable to what can be observed in quiescent air [6]. The ionic wind is higher for a consumed power $P / L=80 \mathrm{~W} / \mathrm{m}$. The maximum found ionic wind addition corresponds to $U_{\text {plasma }} / U_{e}=0.2$ and is around $7 \mathrm{~m} / \mathrm{s}$.

\section{CONCLUDING REMARKS AND OUTLOOK}

In this article, an experimental characterization of DBD plasma actuation on a Blasius boundary layer has been presented. A focus has been made

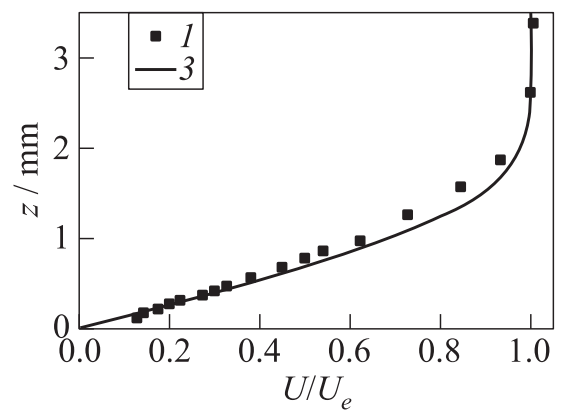

(a)

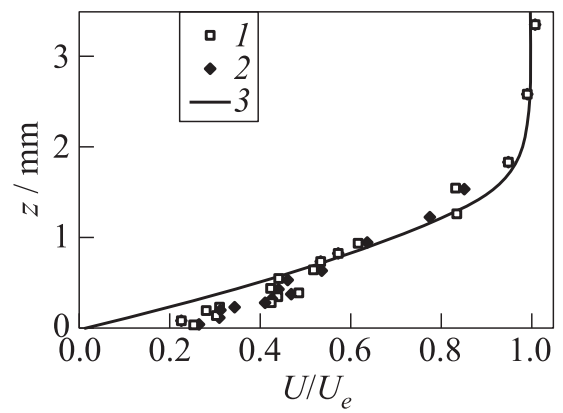

(b)

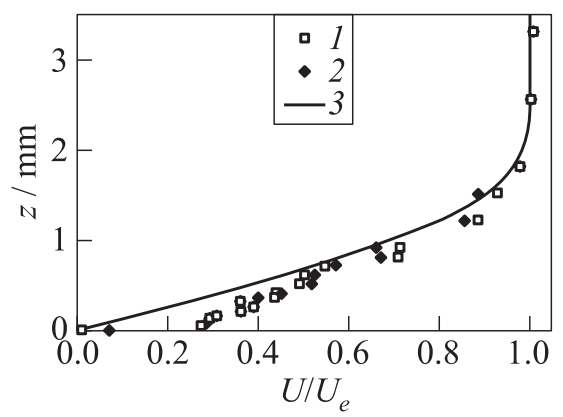

(c)

Figure 14 Mean velocity profiles measured at $x_{\mathrm{DBD}}+4 \mathrm{~mm}(1$ - corrected and 2 - noncorrected) compared to the corresponding Blasius velocity profile (3) for various cases: without actuation $(a)$ and with actuation for $P / L=40(b)$ and $80 \mathrm{~W} / \mathrm{m}(c)$ 


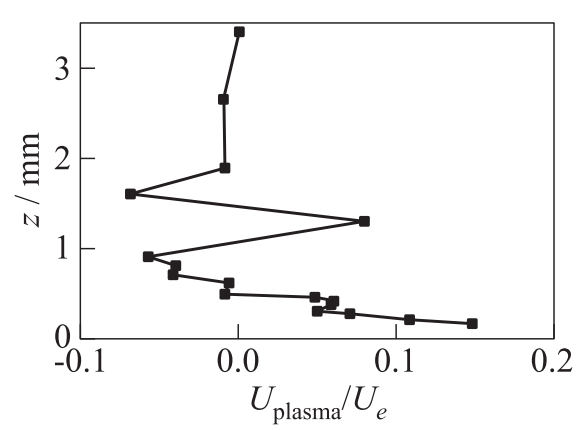

(a)

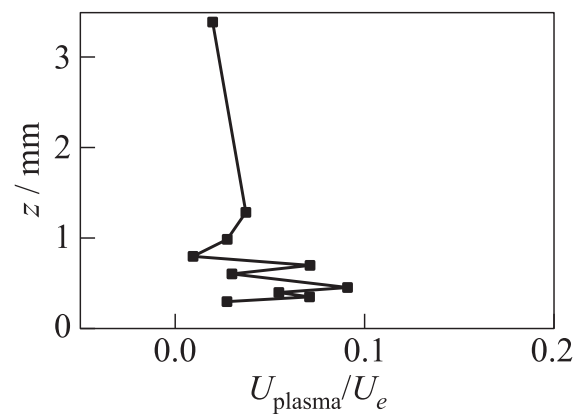

(b)

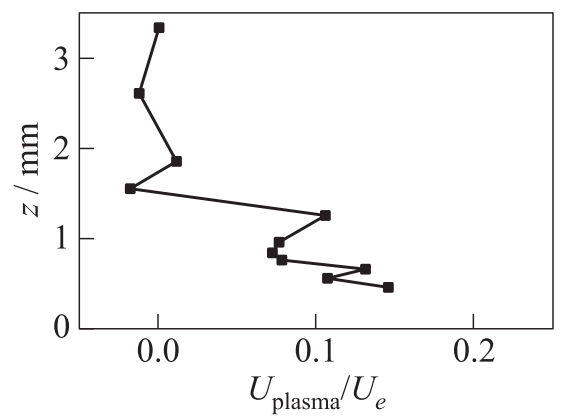

(c)

Figure 15 Added ionic wind at several locations inside and close to the plasma extent for a consumed power $P / L$ $=40 \mathrm{~W} / \mathrm{m}:(a)$ at $x_{\mathrm{DBD}}-2 \mathrm{~mm} ;(b)$ at $x_{\mathrm{DBD}}+2 \mathrm{~mm}$; and $(c)$ at $x_{\mathrm{DBD}}+14 \mathrm{~mm}$

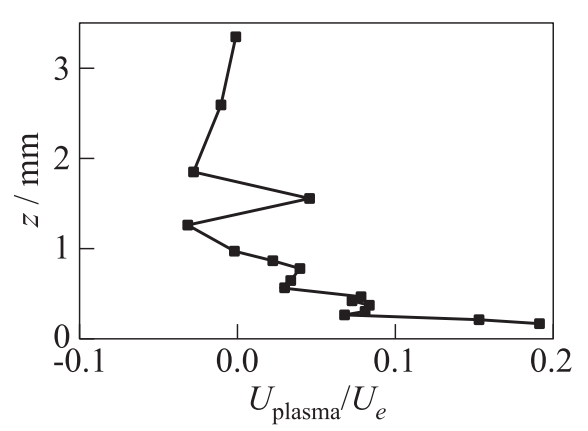

(a)

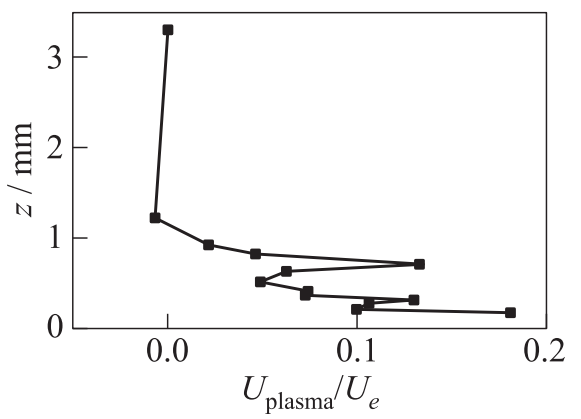

(b)

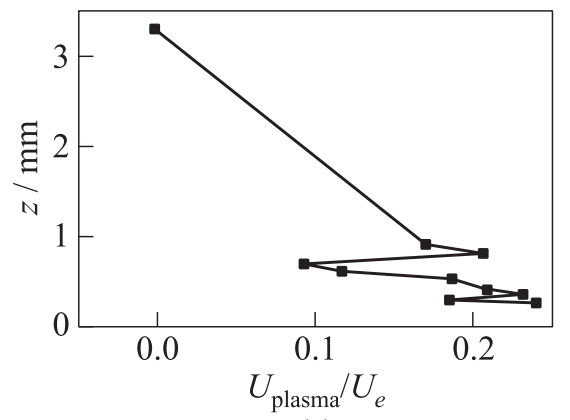

(c)

Figure 16 Added ionic wind at several locations inside and close to the plasma extent for a consumed power $P / L$ $=80 \mathrm{~W} / \mathrm{m}:(a)$ at $x_{\mathrm{DBD}}-2 \mathrm{~mm} ;(b)$ at $x_{\mathrm{DBD}}+2 \mathrm{~mm}$; and $(c)$ at $x_{\mathrm{DBD}}+14 \mathrm{~mm}$ 
on steady actuation by choosing a suita ble input signal frequency: excitation of naturally amplified T-S waves has been avoided. For the presented actuation configurations, a transition delay of at least $30 \mathrm{~mm}$ has been obtained, which confirmed the absence of undesired unsteady actuation effects.

Mean velocity profiles measurements have been performed outside and inside the plasma extent. Outside the plasma extent, mean velocity profiles measured with hot wire anemometry and LDA show a great accordance while DBD actuation is performed. Even if 50 and $100 \mathrm{~mm}$ downstream the actuator, the ionic wind addition is slightly visible, the boundary layer is still under influence of the actuation, which can be seen as the displacement thickness is greater when the actuator is powered. Inside the plasma extent, mean velocity profiles are measured only with LDA. As the seeding particles seem to be influenced by the electric field generated by the actuator, a correction has then been applied to dismiss this effect. The plasma actuation effect can be seen below $z=1 \mathrm{~mm}$ from the wall. The added ionic wind is computed by substracting mean velocity profiles with and without actuation. The maximal measured ionic wind is around $7 \mathrm{~m} / \mathrm{s}$ for a consumed power $P / L=80 \mathrm{~W} / \mathrm{m}$. It has been noticed that the effects of the ionic wind are visible some millimeters upstream the actuators.

More measurements will be done for other free-stream velocities, especially inside the plasma extent. They will allow to inquire about the dependency of the added ionic wind on the free-stream velocity. The vertical component of the velocity will also be studied. These sets of measurements will allow to improve an existing empirical body force model, which will be implemented in a boundary layer code. This model will allow a numerical study of actuation to obtain an optimized actuation configuration with several DBD actuators. This configuration will then be experimentally tested.

\section{ACKNOWLEDGMENTS}

The authors would like to thank Francis Micheli and Estelle Piot for their grateful help with the LDA measurements. Part of this work is supported by the European FP7 Project BUTERFLI (FP7-AAT-2013.8-1-RTD-RUSSIA) program. This work is part of a PhD work which is funded by DGA (Dëlégatin Générale de l'Armement).

\section{REFERENCES}

1. Grundmann, S., and C. Tropea. 2007. Experimental transition delay using glowdischarge plasma actuators. Exp. Fluids 42:653-657. 
2. Joussot, R., R. Weber, A. Leroy, and D. Hong. 2013. Transition control using a single plasma actuator. Int. J. Aerodyn. 3(1/2/3):26-46. doi: 10.1504/IJAD.2013.050912.

3. Séraudie, A., O. Vermeersch, and D. Arnal. 2011. DBD Plasma actuator effect on a 2D model laminar boundary layer. Transition delay under ionic wind effect. 29th AIAA Applied Aerodynamics Conference.

4. Duchmann, A., B. Simon, C. Tropea, and S. Grundmann. 2014. Dielectric barrier discharge plasma actuators for in-flight transition delay. AIAA J. 52(2):358-367.

5. Szulga, N., O. Vermeersch, M. Forte, and G. Casalis. Experimental and numerical study of boudary layer transition control over an airfoil using a DBD plasma actuator. IUTAM_ABCM Symposium on Laminar Turbulent Transition.

6. Forte, M., J. Jolibois, J. Pons, E. Moreau, G. Touchard, and M. Cazalens. 2007. Optimization of a dielectric barrier discharge actuator by stationary and non-stationary measurements of the induced flow velocity: Application to airflow control. Exp. Fluids 43(6):917-928.

7. Mack, L. M. 1977. Transition prediction and linear stability theory. AGARD Conference Proceedings. No. 224.

8. Debien, A., N. Benard, L. David, and E. Moreau. 2012. Unsteady aspect of the electrohydrodynamic force produced by surface dielectric barrier discharge actuators. Appl. Phys. Lett. 100(1):013901. doi: 10.1063/1.3674308. 